\title{
ON HYPERSURFACES INTO RIEMANNIAN SPACES OF CONSTANT SECTIONAL CURVATURE
}

\author{
Antonio CAminha
}

\begin{abstract}
In this paper, we compute $L_{r}\left(S_{r}\right)$ for an isometric immersion $x: M^{n} \rightarrow \bar{M}_{c}^{n+1}$, from an $n$-dimensional Riemannian manifold $M^{n}$ into an $(n+1)$-dimensional Riemannian manifold $\bar{M}_{c}^{n+1}$, of constant sectional curvature $c$. Here, by $L_{r}$ we mean the linearization of the second order differential operator associated to the $(r+1)$-th elementary symmetric function $S_{r+1}$ on the eigenvalues of the second fundamental form $A$ of $x$. The resulting formulae are then applied to study how the behavior of higher-order mean curvature functions of $M^{n}$ influence its geometry.
\end{abstract}

\section{Introduction}

In a seminal paper ([15]), J. Simons computed the Laplacian of the second fundamental form of isometric immersions in spheres, applying the result to get an integral inequality to be satisfied by the squared norm of the second fundamental form $A$ of a minimal oriented hypersurface of the unit sphere $\mathbf{S}^{n+1}$. More specifically, he proved that

$$
\int_{M}|A|^{2}\left(n-|A|^{2}\right) d M \leq 0
$$

what immediately gives a gap theorem concerning the size of the squared norm of $A$ for minimal hypersurfaces of the sphere. In fact, if $0 \leq|A|^{2} \leq n$ for such an immersion, then one has $|A|^{2}=0$ or $n$.

Following Simons' approach and working independently, S. S. Chern, M. do Carmo and S. Kobayashi in [6], and H. B. Lawson in [12], characterized minimal Clifford tori $\mathbf{S}_{r_{1}}^{n_{1}} \times \mathbf{S}_{r_{2}}^{n_{2}}, n_{1}+n_{2}=n, r_{1}=\sqrt{n_{1} / n}, r_{2}=\sqrt{n_{2} / n}$ as the only closed minimal hypersurfaces of the unit sphere $\mathbf{S}^{n+1}$ for which $|A|^{2}=n$. The natural immediate generalization, namely, the study of rigidity properties of constant mean curvature hypersurfaces of the sphere under appropriate constraints on $|A|^{2}$, is due to H. Alencar and M. do Carmo, in [1], still working along the same lines of [15].

2000 Mathematics Subject Classification. Primary 53C21; Secondary 53C20, 53B21.

Received July 6, 2005; revised September 27, 2005. 
A slight variation of this general method for investigating rigidity properties of hypersurfaces in Riemannian spaces of constant sectional curvature appeared in a work of H. Alencar, M. do Carmo and A. G. Colares (see [2]), where the authors obtained a formula for $L_{1}\left(S_{1}\right)$ under the additional hypothesis that $M$ had scalar curvature identically equal to that of the ambient space. This formula was later used by H. Alencar, M. do Carmo and W. Santos (see [3]) to prove a gap theorem for compact orientable hypersurfaces of the unit sphere having scalar curvature equal to 1 . Here, $x: M^{n} \rightarrow \bar{M}_{c}^{n+1}$ is an $n$-dimensional oriented hypersurface of a Riemannian space $\bar{M}$, of constant sectional curvature $c$, and $A$ denotes the second fundamental form of $x$ with respect to a unit normal vector field $N$ globally defined on $M$. For $0 \leq r \leq n, S_{r}$ is the $r$-th elementary symmetric function on the eigenvalues of $A$, and $P_{r}: T M \rightarrow T M$ is the $r$-th Newton transformation on $M$, recursively defined by $P_{0}=I$ and $P_{r}=S_{r} I-A P_{r-1} ; L_{r}$ is the second order differential operator on $M$, given for a smooth $f: M \rightarrow \mathbf{R}$ by

$$
L_{r}(f)=\operatorname{tr}\left(P_{r} \text { Hess } f\right)
$$

Observe that $L_{0}=\Delta$, the Laplacian of $M$.

In this paper, we compute $L_{r}\left(S_{r}\right)$ for isometric immersions of Riemannian manifolds $M$ as hypersurfaces of Riemannian ambient spaces $\bar{M}$, of constant sectional curvature, without additional restrictions (corollary 3.3). We then apply this formula to study how the behavior of higher order mean curvature functions of $M$ influence its shape. We start generalizing (theorem 4.3) the abovementioned gap theorem of Alencar and do Carmo for hypersurfaces of the unit sphere having constant mean or scalar curvature (not necessarily equal to 1). Then we prove a result (theorem 4.5) generalizing Simons' integral inequality for $r$-minimal hypersurfaces of the sphere. The above-mentioned results of Chern, do Carmo, Kobayashi and Lawson, as well as a theorem of J. Hounie and M. L. Leite, allows us to characterize $(r-1)$-minimal Clifford tori $\mathbf{S}_{r_{1}}^{n_{1}} \times \mathbf{S}_{r_{2}}^{n_{2}}$, with $r_{1}^{2}+r_{2}^{2}=1$ and $n_{1}+n_{2}=n$, as the only closed oriented hypersurfaces of the unit sphere $\mathbf{S}^{n+1}$ for which $S_{r}=0, S_{r+1} \neq 0$ and

$$
\operatorname{tr}\left(A^{2} P_{r-1}\right) \operatorname{tr}\left(P_{r-1}\right) \geq \operatorname{tr}\left(A^{2} P_{r-1}\right)^{2} .
$$

We next apply the formula for $L_{r}\left(S_{r}\right)$, together with a theorem of J. L. Barbosa and A. G. Colares giving sufficient conditions for the ellipticity of the operator $L_{r}$, to characterize, in theorem 4.9, geodesic hyperspheres as the only closed orientable hypersurfaces of the standard Riemannian space forms having one constant nonzero higher order mean curvature. Then we show, for the case of hypersurfaces of the unit sphere, how much one can relax the condition of $M$ being contained in an open hemisphere (necessary to apply the theorem of Barbosa and Colares), obtaining a result (theorem 4.11) that also works as a sort of analogue to the gap theorem of Alencar and do Carmo for general $1 \leq r \leq n$. We finish our discussion with some remarks on noncompact complete hypersurfaces of the Euclidean space $\mathbf{R}^{n+1}$, giving, in theorem 4.12, a characterization of $r$-cilinders $\mathbf{S}^{r} \times \mathbf{R}^{n-r}$. 
This paper is organized in the following manner: in section 2 we establish some notation and recall several results needed for further developments. Then, in section 3, we obtain the formula for $L_{r}\left(S_{r}\right)$ as a corollary of the more general computation of $L_{q}\left(S_{r}\right)$. Finally, in section 4, we state and prove the applications referred to in the above paragraph.

\section{Preliminaries}

Unless stated otherwise, we mean by $M^{n}$, or simply $M$, an $n$-dimensional orientable Riemannian manifold, with Riemannian metric $g=\langle$,$\rangle , Levi-Civita$ connection $\nabla$ and curvature tensor $R ; \mathscr{D}(M)$ denotes the commutative ring of smooth real functions on $M$.

2.1. Tensor fields. Let $\phi=\langle T \cdot, \cdot\rangle$ denote an arbitrary 2-tensor on $M$, and $\nabla \phi$ and $\nabla^{2} \phi=\nabla(\nabla \phi)$ be its first and second covariant differentials. For each $V \in \mathscr{X}(M)$, the recipe $\left(\nabla_{V} \phi\right)(X, Y)=(\nabla \phi)(X, Y, V)$ defines another 2-tensor on $M$, called the covariant derivative of $\phi$ in the direction of $V$. If $\nabla_{V} T$ denotes the linear operator associated to $\nabla_{V} \phi$, one has

$$
\left(\nabla_{V} T\right)(X)=\nabla_{V}(T X)-T\left(\nabla_{V} X\right) .
$$

Let $\left\{e_{i}\right\}$ be a moving frame on an open neighborhood $U \subset M$, with coframe $\left\{\omega_{i}\right\}$ and connection 1-forms $\omega_{i j}$. Letting $\phi_{i j}$, $\phi_{i j k}$ and $\phi_{i j k l}$ denote the components of $\phi, \nabla \phi$ e $\nabla^{2} \phi$ with respect to $\left\{e_{i}\right\}$, the following relations take place:

$$
\begin{gathered}
\sum_{k} \phi_{i j k} \omega_{k}=d \phi_{i j}-\sum_{k} \phi_{k j} \omega_{i k}-\sum_{k} \phi_{i k} \omega_{j k} ; \\
\sum_{l} \phi_{i j k l} \omega_{l}=d \phi_{i j k}-\sum_{l} \phi_{l j k} \omega_{i l}-\sum_{l} \phi_{i l k} \omega_{j l}-\sum_{l} \phi_{i j l} \omega_{k l} .
\end{gathered}
$$

The proof of the following lemma can be found in [5].

LEMMA 2.1. Let $\phi$ be a 2-tensor on $M$. With respect to an arbitrary moving frame $\left\{e_{k}\right\}$ on $M$, and letting $R_{i r k l}=R\left(e_{i}, e_{r}, e_{k}, e_{l}\right)$, one has

$$
\phi_{i j k l}-\phi_{i j l k}=-\sum_{r} \phi_{r j} R_{i r k l}-\sum_{r} \phi_{i r} R_{r j l k} .
$$

The following remarks on components of tensors with respect to a given moving frame will be used in the next section.

Remark 2.2. A moving frame $\left\{e_{k}\right\}$ on (an open neighborhood of) $M$ is called geodesic at $p$ when $\left(\nabla_{e_{k}} e_{i}\right)(p)=0$ for all $1 \leq i, k \leq n$, which is in turn equivalent to $\omega_{i j}(p)=0$ for all $1 \leq i, j \leq n$. The usual way to build frames on $M$, geodesic at $p \in M$, is by fixing a normal neighborhood of $p$ and parallel 
transporting the elements of an arbitrary orthonormal basis of $T_{p} M$ along the geodesic rays issuing from $p$. Whenever we speak of a frame on $M$, geodesic at some point $p \in M$, we will always assume that it has been built this way.

Remark 2.3. Note also that, for fixed $1 \leq k \leq n$, the above recipe gives $\left(\nabla_{e_{k}} e_{i}\right)(q)=0$, for every $1 \leq i \leq n$ and every point $q$ along the geodesic ray issuing from $p$ with velocity vector $e_{k}$. Therefore, $\omega_{i j}(q)\left(e_{k}\right)=0$ for all such $i, j$ and $q$, and setting $\phi_{i j ; k}=e_{k}\left(\phi_{i j}\right)$ and $\phi_{i j ; k k}=e_{k}\left(e_{k}\left(\phi_{i j}\right)\right)$ one has, along the geodesic ray issuing from $p$ with velocity vector $e_{k}$,

$$
\phi_{i j k}=\phi_{i j ; k} \quad \text { and } \quad \phi_{i j k k}=\phi_{i j ; k k} \text {. }
$$

The first part of (3) follows from (1), while the second one follows from substituting the first into (2).

Remark 2.4. A 2-tensor $\phi$ on $M$ is Codazzi when $\phi_{i j k}=\phi_{i k j}$ for all $1 \leq i, j, k \leq n$, and with respect to any moving frame $\left\{e_{k}\right\}$ on $M$. If this is the case, changing indices $j$ and $k$ in (2) gives

$$
\phi_{i j k l}=\phi_{i k j l}
$$

for all $1 \leq i, j, k, l \leq n$.

A 2-tensor $\phi$ on $M$ is symmetric if $\phi(X, Y)=\phi(Y, X)$ for all $X, Y \in \mathscr{X}(M)$, or equivalently, when its associated linear operator $T$ is self-adjoint. If $X \in$ $\mathscr{X}(M)$ then $\nabla_{X} \phi$ is symmetric whenever $\phi$ is symmetric, so that $\nabla_{X} T$ is self-adjoint whenever $T$ is self-adjoint. With respect to an arbitrary moving frame $\left\{e_{k}\right\}$ on $M$, the symmetry of $\phi$ is equivalent to $\phi_{i j}=\phi_{j i}$, for all $1 \leq i, j \leq n$. We define the squared norm of a symmetric 2-tensor $\phi$ on $M$ by setting

$$
|\phi|^{2}=\operatorname{tr}\left(T^{2}\right)=\sum_{i, j} \phi_{i j}^{2}
$$

where $\operatorname{tr}$ denotes the trace of its associated linear operator $T$.

2.2. Isometric immersions. Let $x: M^{n} \rightarrow \bar{M}^{n+1}$ denote an isometric immersion from $M^{n}$ into an $(n+1)$-dimensional, oriented Riemannian manifold $\bar{M}^{n+1}$. Also, suppose $M$ oriented by the choice of a unit normal vetor field $N$, and denote by $A$ the corresponding second fundamental form. When $\bar{M}^{n+1}$ has constant sectional curvature $c$, we recall Gauss' and Codazzi's equations: for $W, X, Y, Z \in \mathscr{X}(M)$, one has

$$
\begin{aligned}
\langle R(W, X) Y, Z\rangle= & c[\langle W, Y\rangle\langle X, Z\rangle-\langle W, Z\rangle\langle X, Y\rangle] \\
& +[\langle A W, Y\rangle\langle A X, Z\rangle-\langle A W, Z\rangle\langle A X, Y\rangle]
\end{aligned}
$$

and 


$$
\left(\nabla_{X} A\right) Y=\left(\nabla_{Y} A\right) X .
$$

Note that in this case Codazzi's equation (6) is exactly what it means for the second fundamental form $A$ to be a Codazzi tensor.

Associated to the second fundamental form $A$ of a general isometric immersion $x: M^{n} \rightarrow \bar{M}^{n+1}$ one has $n$ invariants $S_{r}, 1 \leq r \leq n$, given by the equality

$$
\operatorname{det}(t I-A)=\sum_{k=0}^{n}(-1)^{k} S_{k} t^{n-k},
$$

where $S_{0}=1$ by definition. If $p \in M$ and $\left\{e_{k}\right\}$ is a basis of $T_{p} M$ formed by eigenvectors of $A_{p}$, with corresponding eigenvalues $\left\{\lambda_{k}\right\}$, one immediately sees that

$$
S_{r}=\sigma_{r}\left(\lambda_{1}, \ldots, \lambda_{n}\right),
$$

where $\sigma_{r} \in \mathbf{R}\left[X_{1}, \ldots, X_{n}\right]$ is the $r$-th elementary symmetric polynomial on the indeterminates $X_{1}, \ldots, X_{n}$. In particular,

$$
|A|^{2}+2 S_{2}=S_{1}^{2}
$$

The following lemma appears, in a slightly different form, in [2].

Lemma 2.5. Let $x: M^{n} \rightarrow \bar{M}^{n+1}$ be an isometric immersion. If $S_{2}$ is constant on $M$, then

$$
S_{1}^{2}\left(|\nabla A|^{2}-\left|\nabla S_{1}\right|^{2}\right) \geq 2 S_{2}|\nabla A|^{2} .
$$

In particular, if $S_{2} \geq 0$ then $|\nabla A|^{2}-\left|\nabla S_{1}\right|^{2} \geq 0$.

Note also that if $R$ denotes the scalar curvature of $M$, and $\bar{M}$ has constant sectional curvature $c$, it follows from Gauss' equation that

$$
2 S_{2}=n(n-1)(R-c),
$$

so that $S_{2}$ is constant on $M$ if and only if $R$ is constant on $M$.

2.3. Newton transformations. For $0 \leq r \leq n$, one defines the $r$-th Newton operator $P_{r}$ on $M$ by setting $P_{0}=I$ (the identity operator) and, for $1 \leq r \leq n$, via the recursion formulae

$$
P_{r}=S_{r} I-A P_{r-1} .
$$

A trivial induction shows that

$$
P_{r}=S_{r} I-S_{r-1} A+S_{r-2} A^{2}-\cdots+(-1)^{r} A^{r},
$$

from where Cayley-Hamilton theorem gives $P_{n}=0$. Moreover, since $P_{r}$ is a polynomial on $A$ for every $r$, it is also self-adjoint and commutes with $A$. Therefore, all bases of $T_{p} M$ diagonalizing $A$ at $p \in M$ also diagonalize all of the 
$P_{r}$ at $p$. Hence, denoting by $A_{i}$ the restriction of $A$ to $\left\langle e_{i}\right\rangle^{\perp} \subset T_{p} M$, it is easy to see that

$$
\operatorname{det}\left(t I-A_{i}\right)=\sum_{k=0}^{n-1}(-1)^{k} S_{k}\left(A_{i}\right) t^{n-1-k}
$$

where

$$
S_{k}\left(A_{i}\right)=\sum_{\substack{1 \leq j_{1}<\cdots<j_{k} \leq n \\ j_{1}, \ldots, j_{k} \neq i}} \lambda_{j_{1}} \cdots \lambda_{j_{k}}
$$

With the above notations, one can easily prove that $P_{r} e_{i}=S_{r}\left(A_{i}\right) e_{i}$, and also that

(a) $S_{r}\left(A_{i}\right)=S_{r}-\lambda_{i} S_{r-1}\left(A_{i}\right)$.

(b) $\operatorname{tr}\left(P_{r}\right)=\sum_{i=1}^{n} S_{r}\left(A_{i}\right)=(n-r) S_{r}$.

(c) $\operatorname{tr}\left(A P_{r}\right)=\sum_{i=1}^{n} \lambda_{i} S_{r}\left(A_{i}\right)=(r+1) S_{r+1}$.

(d) $\operatorname{tr}\left(A^{2} P_{r}\right)=\sum_{i=1}^{n} \lambda_{i}^{2} S_{r}\left(A_{i}\right)=S_{1} S_{r+1}-(r+2) S_{r+2}$.

Concerning general bases of $T_{p} M$, the following lemma is due to R. Reilly ([14]). For the sake of completeness, and also to establish some notation, we include a short proof of it.

Lemma 2.6. If $\left(h_{i j}\right)$ denotes the matrix of $A$ with respect to a certain orthonormal basis $\beta=\left\{e_{k}\right\}$ of $T_{p} M$, then the matrix $\left(h_{i j}^{r}\right)$ of $P_{r}$ with respect to the same basis is given by

$$
h_{i j}^{r}=\frac{1}{r !} \sum_{i_{k}, j_{k}=1}^{n} \epsilon_{i_{1} \cdots i_{r} i}^{j_{1} \cdots j_{r} j} h_{j_{1} i_{1}} \cdots h_{j_{r} i_{r}}
$$

where

$$
\epsilon_{i_{1} \cdots i_{r}}^{j_{1} \cdots j_{r}}= \begin{cases}\operatorname{sgn}(\sigma), & \text { if the } i_{k} \text { are pairwise distinct and } \\ & \sigma=\left(j_{k}\right) \text { is a permutation of them } \\ 0, & \text { else. }\end{cases}
$$

Proof. Recall that $P_{r}=\sum_{j=0}^{n}(-1)^{j} S_{r-j} A^{j}$, with the coefficients $S_{r-j}$ not depending on the chosen basis of $T_{p} M$. Therefore, it suffices to verify the above formula when $\beta$ diagonalizes $A$ at $p$, with, say, $A e_{k}=\lambda_{k} e_{k}$ for $1 \leq k \leq n$. In this case, the right hand side of (9) sucessively equals

$$
\begin{aligned}
& \frac{1}{r !} \sum_{i_{k}, j_{k}=1}^{n} \epsilon_{i_{1} \cdots i_{r} i}^{j_{1} \cdots j_{r} j} \delta_{j_{1} i_{1}} \cdots \delta_{j_{r} i_{r}} \lambda_{j_{1}} \cdots \lambda_{j_{r}} \\
& =\frac{1}{r !} \sum_{i_{k} \neq i} \epsilon_{i_{1} \cdots i_{r} i}^{i_{1} \cdots i_{r} j} \lambda_{i_{1}} \cdots \lambda_{i_{r}}=\delta_{i j} \sum_{\substack{i_{1}<\cdots<i_{r} \\
i_{k} \neq i}} \lambda_{i_{1}} \cdots \lambda_{i_{r}} \\
& =\delta_{i j} S_{r}\left(A_{i}\right)=\left\langle P_{r} e_{i}, e_{j}\right\rangle=h_{i j}^{r} .
\end{aligned}
$$


We use the above lemma to compute first derivatives of $h_{i j}^{r}$ :

Lemma 2.7. Let $\left\{e_{k}\right\}$ be a moving frame on a neighborhood of $p \in M$, diagonalizing the second fundamental form $A$ at $p$, with $A e_{k}=\lambda_{k} e_{k}$ for $1 \leq$ $k \leq n$. Then, for $1 \leq i, j, k \leq n, i \neq j$, one has at $p$

$$
e_{k}\left(h_{i i}^{r}\right)=\sum_{l \neq i} S_{r-1}\left(A_{i l}\right) h_{l l ; k}
$$

and

$$
e_{k}\left(h_{i j}^{r}\right)=-S_{r-1}\left(A_{i j}\right) h_{i j ; k},
$$

where $A_{i j}$ denotes the restriction of $A$ to $\left\{e_{i}, e_{j}\right\}^{\perp} \subset T_{p} M$.

Proof. Forgetting for the moment the restriction of being $i \neq j$, it follows from (9) that

$$
\begin{aligned}
e_{k}\left(h_{i j}^{r}\right)= & \frac{1}{r !} \sum_{i_{k}, j_{k}=1}^{n} \epsilon_{i_{1} \cdots i_{r} i}^{j_{1} \cdots j_{j} j} h_{j_{1} i_{1} ; k} h_{j_{2} i_{2}} \cdots h_{j_{r} i_{r}}+\cdots \\
& +\frac{1}{r !} \sum_{i_{k}, j_{k}=1}^{n} \epsilon_{i_{1} \cdots i_{r} i}^{j_{1} \cdots j_{r} j} h_{j_{1} i_{1}} \cdots h_{j_{r-1} i_{r-1}} h_{j_{r} i_{r} ; k} .
\end{aligned}
$$

At $p$, the first summand on the right hand side equals

$$
\frac{1}{r !} \sum_{i_{k}, j_{k}=1}^{n} \epsilon_{i_{1} \cdots i_{r} i}^{j_{1} \cdots j_{r} j} \delta_{i_{2} j_{2}} \cdots \delta_{i_{r} j_{r}} h_{j_{1} i_{1} ; k} \lambda_{i_{2}} \cdots \lambda_{i_{r}}=\frac{1}{r !} \sum_{i_{k}, j_{1}=1}^{n} \epsilon_{i_{1} i_{2} \cdots i_{r} i}^{j_{1} i_{2} \cdots i_{i_{1} j} j} h_{j_{1} i_{1} ; k} \lambda_{i_{2}} \cdots \lambda_{i_{r}} .
$$

Now, consider two separate cases: for $i=j$,

$$
\begin{aligned}
(13) & =\frac{1}{r !} \sum_{i_{k}, j_{1}=1}^{n} \epsilon_{i_{1} i_{2} \cdots i_{r} i}^{j_{1} i_{2} \cdots i_{r} i} h_{j_{1} i_{1} ; k} \lambda_{i_{2}} \cdots \lambda_{i_{r}} \\
& =\frac{1}{r !} \sum_{1 \leq i_{k} \leq n} \epsilon_{i_{1} i_{2} \cdots i_{r} i}^{i_{1} i_{2} \cdots i_{r} i} h_{i_{1} i_{1} ; k} \lambda_{i_{2}} \cdots \lambda_{i_{r}} \\
& =\frac{1}{r} \sum_{l \neq i} \sum_{\substack{i_{2}<\cdots<i_{r} \\
i_{k} \neq i, l}} h_{l l ; k} \lambda_{i_{2}} \cdots \lambda_{i_{r}}=\frac{1}{r} \sum_{l \neq i} S_{r-1}\left(A_{i l}\right) h_{l l ; k} .
\end{aligned}
$$

Since the same is true for all other summands in (12), one gets (10). For $i \neq j$, it follows from the definition of $\epsilon_{i_{1} i_{2} \cdots i_{i} i}^{j_{1} i_{2} \cdots i_{r} j}$ that 


$$
\begin{aligned}
(13) & =\frac{1}{r !} \sum_{i_{k} \neq i, j} \epsilon_{j i_{2} \cdots i_{r} i}^{i i_{2} \cdots i_{r} j} h_{i j ; k} \lambda_{i_{2}} \cdots \lambda_{i_{r}} \\
& =-\frac{1}{r} \sum_{\substack{i_{k} \neq i, j \\
i_{2}<\cdots<i_{r}}} h_{i j ; k} \lambda_{i_{2}} \cdots \lambda_{i_{r}}=-\frac{1}{r} S_{r-1}\left(A_{i j}\right) h_{i j ; k},
\end{aligned}
$$

so that (11) now follows from (12).

In the sequel, we will need the following proposition. Item (a) is essentially the content of lemma 1.1 and equation (1.3) in [10], while item (b) is quoted as proposition 1.5 in [11].

Proposition 2.8. Let $x: M^{n} \rightarrow \bar{M}^{n+1}$ be an isometric immersion, and $1 \leq r<n, p \in M$.

(a) If $S_{r}(p)=0$, then $P_{r-1}$ is semi-definite at $p$.

(b) If $S_{r}(p)=0$ and $S_{r+1}(p) \neq 0$, then $P_{r-1}$ is definite at $p$.

Finally, for $0 \leq r \leq n$, let $L_{r}: \mathscr{D}(M) \rightarrow \mathscr{D}(M)$ be the second order differential operator given by

$$
L_{r}(f)=\operatorname{tr}\left(P_{r} \text { Hess } f\right) \text {. }
$$

When $\bar{M}^{n+1}$ has constant seccional curvature, it was proved by H. Rosenberg in [13] that

$$
L_{r}(f)=\operatorname{div}\left(P_{r} \nabla f\right)
$$

where div stands for the divergence of a vector field on $M$. Thus, for $f, g \in \mathscr{D}(M)$, it follows from the properties of the divergence of vector fields that

$$
L_{r}(f g)=f L_{r}(g)+g L_{r}(f)+2\left\langle P_{r} \nabla f, \nabla g\right\rangle .
$$

\section{The formula for $L_{r}\left(S_{r}\right)$}

As in the previous section, $x: M^{n} \rightarrow \bar{M}_{c}^{n+1}$ denotes an isometric immersion between oriented Riemannian manifolds, and $A$ denotes the corresponding second fundamental form.

Proposition 3.1. Let $x: M^{n} \rightarrow \bar{M}_{c}^{n+1}$ be an isometric immersion, and $0 \leq q<n, 0<r<n$. If $\left\{e_{k}\right\}$ is any orthonormal frame on $M$, then

$$
\begin{aligned}
L_{q}\left(S_{r}\right)= & L_{r-1}\left(S_{q+1}\right) \\
& +\sum_{k} \operatorname{tr}\left\{\left[P_{q}\left(\nabla_{e_{k}} P_{r-1}\right)-P_{r-1}\left(\nabla_{e_{k}} P_{q}\right)\right]\left(\nabla_{e_{k}} A\right)\right\} \\
& +c\left[\operatorname{tr}\left(A P_{r-1}\right) \operatorname{tr}\left(P_{q}\right)-\operatorname{tr}\left(P_{r-1}\right) \operatorname{tr}\left(A P_{q}\right)\right] \\
& +\operatorname{tr}\left(A^{2} P_{r-1}\right) \operatorname{tr}\left(A P_{q}\right)-\operatorname{tr}\left(A P_{r-1}\right) \operatorname{tr}\left(A^{2} P_{q}\right) .
\end{aligned}
$$


Proof. First of all, observe that the validity of (14) does not depend on the particular chosen frame $\left\{e_{k}\right\}$. So, let $p \in M$ and $\left\{e_{k}\right\}$ be a moving frame on a neighborhood $U \subset M$ of $p$, diagonalizing $A$ at $p$, with $A e_{k}=\lambda_{k} e_{k}$ for $1 \leq k \leq n$. Denote respectively by $h_{i j}$ and $h_{i j}^{r}$ the components of $A$ and $P_{r}$ with respect to such a frame. It follows from equation (9) that

$$
\begin{aligned}
h_{i i}^{r} & =\frac{1}{r !} \sum_{i_{k}, j_{k}=1}^{n} \epsilon_{i_{1} \cdots i_{r} i}^{j_{1} \cdots j_{r} i} h_{j_{1} i_{1}} \cdots h_{j_{r} i_{r}} \\
& =\frac{1}{r !} \sum_{i_{k} \neq i, \sigma=\left(j_{k}\right)} \operatorname{sgn}(\sigma) h_{j_{1} i_{1}} \cdots h_{j_{r} i_{r}} \\
& =\sum_{\substack{i_{1}<\cdots<i_{r} \\
i_{k} \neq i}} \sum_{\sigma=\left(j_{k}\right)} \operatorname{sgn}(\sigma) h_{j_{1} i_{1}} \cdots h_{j_{r} i_{r}} \\
& =\sum_{\substack{i_{1}<\cdots<i_{r} \\
i_{k} \neq i}} A\left(c_{i_{1}}, \ldots, c_{i_{r}}\right),
\end{aligned}
$$

where by $A\left(c_{i_{1}}, \ldots, c_{i_{r}}\right)$ we mean the $r \times r$ determinant minor of $A$, obtained by choosing lines and columns of $A$ with indices $i_{1}<\cdots<i_{r}$. Hence,

$$
\begin{aligned}
S_{r} & =\frac{1}{n-r} \operatorname{tr}\left(P_{r}\right)=\frac{1}{n-r} \sum_{i} \sum_{\substack{i_{1}<\cdots<i_{r} \\
i_{k} \neq i}} A\left(c_{i_{1}}, \ldots, c_{i_{r}}\right) \\
& =\sum_{i_{1}<\cdots<i_{r}} A\left(c_{i_{1}}, \ldots, c_{i_{r}}\right)
\end{aligned}
$$

for once one has chosen $1 \leq i_{1}<\cdots<i_{r} \leq n$, there will be left $n-r$ possible choices for $i$ in $\{1, \ldots, n\}$. Since determinants are multilinear functions of their columns, one gets

$$
e_{k}\left(S_{r}\right)=\sum_{i_{1}<\cdots<i_{r}}\left[A\left(c_{i_{1} ; k}, c_{i_{2}}, \ldots, c_{i_{r}}\right)+\cdots+A\left(c_{i_{1}}, \ldots, c_{i_{r-1}}, c_{i_{r} ; k}\right)\right]
$$

on $U$. At $p$, one has

$$
A\left(c_{i_{1} ; k}, c_{i_{2}}, \ldots, c_{i_{r}}\right)=\left|\begin{array}{cccc}
h_{i_{1} i_{1} ; k} & 0 & \cdots & 0 \\
h_{i_{2} i_{1} ; k} & \lambda_{i_{2}} & \cdots & 0 \\
\vdots & \vdots & \vdots & \vdots \\
h_{i_{r} i_{1} ; k} & 0 & \cdots & \lambda_{i_{r}}
\end{array}\right|=h_{i_{1} i_{1} ; k} \lambda_{i_{2}} \cdots \lambda_{i_{r}},
$$

and analogously for the remaining summands, so that

$$
\begin{aligned}
e_{k}\left(S_{r}\right) & =\sum_{i_{1}<\cdots<i_{r}}\left(h_{i_{1} i_{1} ; k} \lambda_{i_{2}} \cdots \lambda_{i_{r}}+\cdots+\lambda_{i_{1}} \cdots \lambda_{i_{r-1}} h_{i_{r} i_{r} ; k}\right) \\
& =\sum_{i=1}^{n} h_{i i ; k} S_{r-1}\left(A_{i}\right) .
\end{aligned}
$$


The last equality follows from the fact that, for fixed $1 \leq i \leq n, h_{i i ; k}$ appears in the above sum together with all products $\lambda_{j_{1}} \ldots \lambda_{j_{r-1}}$, with $j_{1}, \ldots, j_{r-1} \neq i$ (note that the above formula for $e_{k}\left(S_{r}\right)$ could have been obtained directly from (10). This alternative approach was chosen to ease, in what comes next, the computation of second derivatives).

To compute second derivatives, suppose further $\left\{e_{k}\right\}$ geodesic at $p$. It follows from (16) that

$$
\begin{aligned}
e_{k}\left(e_{k}\left(S_{r}\right)\right)= & \sum_{i_{1}<\cdots<i_{r}}\left[A\left(c_{i_{1} ; k k}, c_{i_{2}}, \ldots, c_{i_{r}}\right)+\cdots+A\left(c_{i_{1}}, \ldots, c_{i_{r-1}}, c_{i_{r} ; k k}\right)\right] \\
& +\sum_{s \neq t} \sum_{i_{1}<\cdots<i_{r}} A\left(c_{i_{1}}, \ldots, c_{i_{s} ; k}, \ldots, c_{i_{t} ; k}, \ldots, c_{i_{r}}\right),
\end{aligned}
$$

and one gets at $p$

$$
\begin{aligned}
e_{k}\left(e_{k}\left(S_{r}\right)\right)= & \sum_{i_{1}<\cdots<i_{r}}\left(h_{i_{1} i_{1} ; k k} \lambda_{i_{2}} \cdots \lambda_{i_{r}}+\cdots+\lambda_{i_{1}} \cdots \lambda_{i_{r-1}} h_{i_{r} i_{r} ; k k}\right) \\
& +\sum_{\substack{i_{1}<\cdots<i_{r} \\
s \neq t}}\left(h_{i_{s} i_{s} ; k} h_{i_{t} i_{i} ; k}-h_{i_{s} i_{i} ; k} h_{i_{t} i_{s} ; k}\right) \lambda_{i_{1}} \cdots \hat{\lambda}_{i_{s}} \cdots \hat{\lambda}_{i_{t}} \cdots \lambda_{i_{r}} .
\end{aligned}
$$

Grouping equal occurrences of $(r-2)$-tuples $i_{1}<\cdots<i_{r-2}$ in the last expression above, $e_{k}\left(e_{k}\left(S_{r}\right)\right)$ equals

$$
\sum_{i} \sum_{\substack{i_{1}<\cdots<i_{r-1} \\ i_{k} \neq i}} h_{i i ; k k} \lambda_{i_{1}} \cdots \lambda_{i_{r-1}}+\sum_{i \neq j} \sum_{\substack{i \\ i_{1}<\cdots<i_{r-2} \\ i_{k} \neq i, j}}\left[h_{i i ; k} h_{j j ; k}-h_{i j ; k}^{2}\right] \lambda_{i_{1}} \cdots \lambda_{i_{r-2}},
$$

and finally

$$
e_{k}\left(e_{k}\left(S_{r}\right)\right)=\sum_{i} S_{r-1}\left(A_{i}\right) h_{i i ; k k}+\sum_{i \neq j} S_{r-2}\left(A_{i j}\right)\left[h_{i i ; k} h_{j j ; k}-h_{i j ; k}^{2}\right] .
$$

Therefore, we get at $p$

$$
\begin{aligned}
L_{q}\left(S_{r}\right)= & \operatorname{tr}\left(P_{q} \operatorname{Hess}\left(S_{r}\right)\right)=\sum_{k=1}^{n} S_{q}\left(A_{k}\right) e_{k}\left(e_{k}\left(S_{r}\right)\right) \\
= & \sum_{i, k} S_{q}\left(A_{k}\right) S_{r-1}\left(A_{i}\right) h_{i i ; k k}+\sum_{\substack{i, j, k \\
i \neq j}} S_{q}\left(A_{k}\right) S_{r-2}\left(A_{i j}\right)\left[h_{i i ; k} h_{j j ; k}-h_{i j ; k}^{2}\right] \\
= & \sum_{i} S_{r-1}\left(A_{i}\right) L_{q}\left(h_{i i}\right)+\sum_{\substack{i, j, k \\
i \neq j}} S_{q}\left(A_{k}\right) S_{r-2}\left(A_{i j}\right) h_{i i ; k} h_{j j ; k} \\
& -\sum_{\substack{i, j, k \\
i \neq j}} S_{q}\left(A_{k}\right) S_{r-2}\left(A_{i j}\right) h_{i j ; k}^{2} .
\end{aligned}
$$


Lemma 2.1, as well as the remarks on commutation of indices in geodesic frames made right after it, allow one to conclude that, at $p$,

$$
\begin{aligned}
& \sum_{i} S_{r-1}\left(A_{i}\right) L_{q}\left(h_{i i}\right) \\
&=\sum_{i, k} S_{r-1}\left(A_{i}\right) S_{q}\left(A_{k}\right) h_{i i k k}=\sum_{i, k} S_{r-1}\left(A_{i}\right) S_{q}\left(A_{k}\right) h_{i k i k} \\
&=\sum_{i, k} S_{r-1}\left(A_{i}\right) S_{q}\left(A_{k}\right)\left(h_{i k i k}-h_{i k k i}+h_{i k k i}-h_{k k i i}+h_{k k i i}\right) \\
&=\sum_{i, k} S_{r-1}\left(A_{i}\right) S_{q}\left(A_{k}\right)\left(h_{i k i k}-h_{i k k i}\right)+\sum_{i, k} S_{r-1}\left(A_{i}\right) S_{q}\left(A_{k}\right) h_{k k i i} \\
&=-\sum_{i, j, k} S_{r-1}\left(A_{i}\right) S_{q}\left(A_{k}\right)\left(h_{j k} R_{i j i k}+h_{i j} R_{j k k i}\right)+\sum_{i, k} S_{r-1}\left(A_{i}\right) S_{q}\left(A_{k}\right) h_{k k i i} \\
&=-\sum_{i, k} S_{r-1}\left(A_{i}\right) S_{q}\left(A_{k}\right) \lambda_{k} R_{i k i k}-\sum_{i, k} S_{r-1}\left(A_{i}\right) S_{q}\left(A_{k}\right) \lambda_{i} R_{i k k i} \\
&+\sum_{k} S_{q}\left(A_{k}\right) L_{r-1}\left(h_{k k}\right) .
\end{aligned}
$$

Now, write $r-1$ in place of $q$ and $q+1$ in place of $r$ in relation (18) to get

$$
\begin{aligned}
L_{r-1}\left(S_{q+1}\right)= & \sum_{i} S_{q}\left(A_{i}\right) L_{r-1}\left(h_{i i}\right)+\sum_{\substack{i, j, k \\
i \neq j}} S_{r-1}\left(A_{k}\right) S_{q-1}\left(A_{i j}\right) h_{i i ; k} h_{j j ; k} \\
& -\sum_{\substack{i, j, k \\
i \neq j}} S_{r-1}\left(A_{k}\right) S_{q-1}\left(A_{i j}\right) h_{i j ; k}^{2} .
\end{aligned}
$$

Substituting the result of (19) into (20), we arrive at

$$
\begin{aligned}
L_{r-1}\left(S_{q+1}\right)= & \sum_{i} S_{r-1}\left(A_{i}\right) L_{q}\left(h_{i i}\right)+\sum_{i, k} S_{r-1}\left(A_{i}\right) S_{q}\left(A_{k}\right) \lambda_{k} R_{i k i k} \\
& +\sum_{i, k} S_{r-1}\left(A_{i}\right) S_{q}\left(A_{k}\right) \lambda_{i} R_{i k k i}+\sum_{\substack{i, j, k \\
i \neq j}} S_{r-1}\left(A_{k}\right) S_{q-1}\left(A_{i j}\right) h_{i i k k} h_{j j ; k} \\
& -\sum_{\substack{i, j, k \\
i \neq j}} S_{r-1}\left(A_{k}\right) S_{q-1}\left(A_{i j}\right) h_{i j ; k}^{2} .
\end{aligned}
$$

Finally, subtracting (21) from (18) gives

$$
\begin{aligned}
L_{q}\left(S_{r}\right)= & L_{r-1}\left(S_{q+1}\right)-\sum_{i, k} S_{r-1}\left(A_{i}\right) S_{q}\left(A_{k}\right) \lambda_{k} R_{i k i k} \\
& -\sum_{i, k} S_{r-1}\left(A_{i}\right) S_{q}\left(A_{k}\right) \lambda_{i} R_{i k k i}+\sum_{\substack{i, j, k \\
i \neq j}} S_{q}\left(A_{k}\right) S_{r-2}\left(A_{i j}\right) h_{i i ; k} h_{j j ; k}
\end{aligned}
$$




$$
\begin{aligned}
& -\sum_{\substack{i, j, k \\
i \neq j}} S_{q}\left(A_{k}\right) S_{r-2}\left(A_{i j}\right) h_{i j ; k}^{2}-\sum_{\substack{i, j, k \\
i \neq j}} S_{r-1}\left(A_{k}\right) S_{q-1}\left(A_{i j}\right) h_{i i ; k} h_{j j ; k} \\
& +\sum_{\substack{i, j, k \\
i \neq j}} S_{r-1}\left(A_{k}\right) S_{q-1}\left(A_{i j}\right) h_{i j ; k}^{2} \cdot
\end{aligned}
$$

In order to better examine the summands at the right hand side of (22), let

$$
I=\sum_{i, k} S_{r-1}\left(A_{i}\right) S_{q}\left(A_{k}\right) \lambda_{k} R_{i k i k}, \quad I I=\sum_{i, k} S_{r-1}\left(A_{i}\right) S_{q}\left(A_{k}\right) \lambda_{i} R_{i k k i} .
$$

Using Gauss' equation, one gets

$$
\begin{aligned}
I= & \sum_{i, k}\left\langle R\left(P_{r-1} e_{i}, P_{q} e_{k}\right) e_{i}, A e_{k}\right\rangle \\
= & c \sum_{i, k}\left[\left\langle P_{r-1} e_{i}, e_{i}\right\rangle\left\langle P_{q} e_{k}, A e_{k}\right\rangle-\left\langle P_{r-1} e_{i}, A e_{k}\right\rangle\left\langle P_{q} e_{k}, e_{i}\right\rangle\right] \\
& +\sum_{i, k}\left[\left\langle A P_{r-1} e_{i}, e_{i}\right\rangle\left\langle A P_{q} e_{k}, A e_{k}\right\rangle-\left\langle A P_{r-1} e_{i}, A e_{k}\right\rangle\left\langle A P_{q} e_{k}, e_{i}\right\rangle\right] \\
= & c\left[\operatorname{tr}\left(P_{r-1}\right) \operatorname{tr}\left(A P_{q}\right)-\sum_{k}\left\langle A P_{r-1} e_{k}, P_{q} e_{k}\right\rangle\right] \\
& +\operatorname{tr}\left(A P_{r-1}\right) \operatorname{tr}\left(A^{2} P_{q}\right)-\sum_{k}\left\langle A^{2} P_{r-1} e_{k}, A P_{q} e_{k}\right\rangle \\
= & c\left[\operatorname{tr}\left(P_{r-1}\right) \operatorname{tr}\left(A P_{q}\right)-\operatorname{tr}\left(A P_{r-1} P_{q}\right)\right]+\operatorname{tr}\left(A P_{r-1}\right) \operatorname{tr}\left(A^{2} P_{q}\right)-\operatorname{tr}\left(A^{3} P_{r-1} P_{q}\right)
\end{aligned}
$$

and

$$
\begin{aligned}
I I= & \sum_{i, k}\left\langle R\left(A e_{i}, P_{q} e_{k}\right) e_{k}, P_{r-1} e_{i}\right\rangle \\
= & c \sum_{i, k}\left[\left\langle A e_{i}, e_{k}\right\rangle\left\langle P_{q} e_{k}, P_{r-1} e_{i}\right\rangle-\left\langle A e_{i}, P_{r-1} e_{i}\right\rangle\left\langle P_{q} e_{k}, e_{k}\right\rangle\right] \\
& +\sum_{i, k}\left[\left\langle A^{2} e_{i}, e_{k}\right\rangle\left\langle A P_{q} e_{k}, P_{r-1} e_{i}\right\rangle-\left\langle A^{2} e_{i}, P_{r-1} e_{i}\right\rangle\left\langle A P_{q} e_{k}, e_{k}\right\rangle\right] \\
= & c\left[\sum_{k}\left\langle A e_{k}, P_{r-1} P_{q} e_{k}\right\rangle-\operatorname{tr}\left(A P_{r-1}\right) \operatorname{tr}\left(P_{q}\right)\right] \\
& +\sum_{k}\left\langle A^{2} e_{k}, A P_{r-1} P_{q} e_{k}\right\rangle-\operatorname{tr}\left(A^{2} P_{r-1}\right) \operatorname{tr}\left(A P_{q}\right) \\
= & c\left[\operatorname{tr}\left(A P_{r-1} P_{q}\right)-\operatorname{tr}\left(A P_{r-1}\right) \operatorname{tr}\left(P_{q}\right)\right]+\operatorname{tr}\left(A^{3} P_{r-1} P_{q}\right)-\operatorname{tr}\left(A^{2} P_{r-1}\right) \operatorname{tr}\left(A P_{q}\right) .
\end{aligned}
$$

On the other hand, letting 


$$
I I I=\sum_{\substack{i, j, k \\ i \neq j}} S_{q}\left(A_{k}\right) S_{r-2}\left(A_{i j}\right) h_{i i ; k} h_{j j ; k}-\sum_{\substack{i, j, k \\ i \neq j}} S_{q}\left(A_{k}\right) S_{r-2}\left(A_{i j}\right) h_{i j ; k}^{2}
$$

and

$$
I V=\sum_{\substack{i, j, k \\ i \neq j}} S_{r-1}\left(A_{k}\right) S_{q-1}\left(A_{i j}\right) h_{i i ; k} h_{j j ; k}-\sum_{\substack{i, j, k \\ i \neq j}} S_{r-1}\left(A_{k}\right) S_{q-1}\left(A_{i j}\right) h_{i j ; k}^{2},
$$

it follows from lemma 2.7 that, at $p$,

$$
\begin{aligned}
\sum_{\substack{i, j, k \\
i \neq j}} S_{q}\left(A_{k}\right) S_{r-2}\left(A_{i j}\right) h_{i i ; k} h_{j j ; k} & =\sum_{i, k} S_{q}\left(A_{k}\right) h_{i i ; k} \sum_{j \neq i} S_{r-2}\left(A_{i j}\right) h_{j j ; k} \\
& =\sum_{i, k} S_{q}\left(A_{k}\right) h_{i i, k} e_{k}\left(h_{i i}^{r-1}\right)
\end{aligned}
$$

and

$$
-\sum_{\substack{i, j, k \\ i \neq j}} S_{q}\left(A_{k}\right) S_{r-2}\left(A_{i j}\right) h_{i j ; k}^{2}=\sum_{\substack{i, j, k \\ i \neq j}} S_{q}\left(A_{k}\right) h_{i j ; k} e_{k}\left(h_{i j}^{r-1}\right) .
$$

Adding these two relations, one gets

$$
I I I=\sum_{i, j, k} S_{q}\left(A_{k}\right) e_{k}\left(h_{i j}^{r-1}\right) h_{i j ; k}=\sum_{k} \operatorname{tr}\left[P_{q}\left(\nabla_{e_{k}} P_{r-1}\right)\left(\nabla_{e_{k}} A\right)\right] .
$$

Again from lemma 2.7, one has at $p$

$$
\begin{aligned}
\sum_{\substack{i, j, k \\
i \neq j}} S_{r-1}\left(A_{k}\right) S_{q-1}\left(A_{i j}\right) h_{i i ; k} h_{j j ; k} & =\sum_{i, k} S_{r-1}\left(A_{k}\right) h_{i i ; k} \sum_{j \neq i} S_{q-1}\left(A_{i j}\right) h_{j j ; k} \\
& =\sum_{i, k} S_{r-1}\left(A_{k}\right) h_{i i ; k} e_{k}\left(h_{i i}^{q}\right)
\end{aligned}
$$

and

$$
-\sum_{\substack{i, j, k \\ i \neq j}} S_{r-1}\left(A_{k}\right) S_{q-1}\left(A_{i j}\right) h_{i j ; k}^{2}=\sum_{\substack{i, j, k \\ i \neq j}} S_{r-1}\left(A_{k}\right) h_{i j ; k} e_{k}\left(h_{i j}^{q}\right)
$$

so that

$$
I V=\sum_{i, j, k} S_{r-1}\left(A_{k}\right) e_{k}\left(h_{i j}^{q}\right) h_{i j ; k}=\sum_{k} \operatorname{tr}\left[P_{r-1}\left(\nabla_{e_{k}} P_{q}\right)\left(\nabla_{e_{k}} A\right)\right] .
$$

It now suffices to substitute the expressions for $I, I I, I I I$ and $I V$ into (22). 
As a byproduct of the computations in the proof of the previous proposition, we get a proof for the following well-known Then

Lemma 3.2. Let $x: M^{n} \rightarrow \bar{M}_{c}^{n+1}$ be an isometric immersion, and $V \in \mathscr{X}(M)$.

$$
\operatorname{tr}\left(P_{r-1}\left(\nabla_{V} A\right)\right)=V\left(S_{r}\right)
$$

Proof. Let $p \in M$, and $\left\{e_{k}\right\}$ be a moving frame on a neighborhood of $p \in M$, geodesic at $p$ and such that, at $p, A e_{k}=\lambda_{k} e_{k}$ for $1 \leq k \leq n$. Since both sides of (23) are linear on $V$, it suffices to prove that $\operatorname{tr}\left(P_{r-1}\left(\nabla_{e_{k}} A\right)\right)=$ $e_{k}\left(S_{r}\right)$.

$$
\begin{aligned}
\operatorname{tr}\left(P_{r-1}\left(\nabla_{e_{k}} A\right)\right) & =\sum_{i=1}^{n}\left\langle P_{r-1}\left(\nabla_{e_{k}} A\right) e_{i}, e_{i}\right\rangle=\sum_{i=1}^{n} S_{r-1}\left(A_{i}\right)\left\langle\left(\nabla_{e_{k}} A\right) e_{i}, e_{i}\right\rangle \\
& =\sum_{i=1}^{n} S_{r-1}\left(A_{i}\right) h_{i i k} .
\end{aligned}
$$

Since the frame is geodesic at $p$, we have $h_{i i k}=h_{i i ; k}$ at $p$, and (17) gives the desired result.

COROllary 3.3. Let $x: M^{n} \rightarrow \bar{M}_{c}^{n+1}$ be an isometric immersion, and $0<r \leq n$. Then

$$
\begin{aligned}
L_{r}\left(S_{r}\right)= & L_{r-1}\left(S_{r+1}\right)+S_{r}\left[\Delta S_{r}-L_{r-1}\left(S_{1}\right)\right] \\
& +\sum_{k}\left|P_{r-1} \nabla_{e_{k}} A\right|^{2}-\left|\nabla S_{r}\right|^{2} \\
& +\operatorname{tr}\left(A P_{r-1}\right)\left\{S_{r}\left(|A|^{2}-c n\right)-\left[\operatorname{tr}\left(A^{2} P_{r}\right)-c \operatorname{tr}\left(P_{r}\right)\right]\right\} \\
& -\operatorname{tr}\left(A^{2} P_{r-1}\right)\left[\operatorname{tr}\left(A^{2} P_{r-1}\right)-c \operatorname{tr}\left(P_{r-1}\right)\right],
\end{aligned}
$$

where $\left\{e_{k}\right\}$ is any orthonormal frame on $M$, or still

$$
\begin{aligned}
L_{r}\left(S_{r}\right)= & L_{r-1}\left(S_{r+1}\right)+S_{r}\left[\Delta S_{r}-L_{r-1}\left(S_{1}\right)\right]+\sum_{k}\left|P_{r-1} \nabla_{e_{k}} A\right|^{2}-\left|\nabla S_{r}\right|^{2} \\
& +\frac{1}{2} \sum_{i, j} S_{r-1}\left(A_{i}\right) S_{r-1}\left(A_{j}\right)\left(\lambda_{i}-\lambda_{j}\right)^{2} K_{M}\left(\sigma_{i j}\right),
\end{aligned}
$$

at $p \in M$, where $\left\{e_{k}\right\}$ is an orthonormal frame on $M$, diagonalizing $A$ at $p$, with $A e_{k}=\lambda_{k} e_{k}$ at $p$, and $\sigma_{i j}$ denotes the 2-dimensional subspace of $T_{p} M$ generated by $e_{i}$ and $e_{j}$.

Proof. It follows from proposition 3.1 that 


$$
\begin{aligned}
L_{r}\left(S_{r}\right)= & L_{r-1}\left(S_{r+1}\right)+\sum_{k} \operatorname{tr}\left\{\left[P_{r}\left(\nabla_{e_{k}} P_{r-1}\right)-P_{r-1}\left(\nabla_{e_{k}} P_{r}\right)\right]\left(\nabla_{e_{k}} A\right)\right\} \\
& +c\left[\operatorname{tr}\left(A P_{r-1}\right) \operatorname{tr}\left(P_{r}\right)-\operatorname{tr}\left(P_{r-1}\right) \operatorname{tr}\left(A P_{r}\right)\right] \\
& +\operatorname{tr}\left(A^{2} P_{r-1}\right) \operatorname{tr}\left(A P_{r}\right)-\operatorname{tr}\left(A P_{r-1}\right) \operatorname{tr}\left(A^{2} P_{r}\right),
\end{aligned}
$$

where $\left\{e_{k}\right\}$ is any orthonormal frame on $M$. Making

$$
T_{k}=\left[P_{r}\left(\nabla_{e_{k}} P_{r-1}\right)-P_{r-1}\left(\nabla_{e_{k}} P_{r}\right)\right]\left(\nabla_{e_{k}} A\right),
$$

we get

$$
\begin{aligned}
T_{k}= & {\left[\left(S_{r} I-A P_{r-1}\right)\left(\nabla_{e_{k}} P_{r-1}\right)-P_{r-1}\left(\nabla_{e_{k}}\left(S_{r} I-A P_{r-1}\right)\right]\left(\nabla_{e_{k}} A\right)\right.} \\
= & S_{r}\left(\nabla_{e_{k}} P_{r-1}\right)\left(\nabla_{e_{k}} A\right)-A P_{r-1}\left(\nabla_{e_{k}} P_{r-1}\right)\left(\nabla_{e_{k}} A\right) \\
& -P_{r-1}\left[e_{k}\left(S_{r}\right) I-\left(\nabla_{e_{k}} A\right) P_{r-1}-A\left(\nabla_{e_{k}} P_{r-1}\right)\right]\left(\nabla_{e_{k}} A\right) \\
= & S_{r}\left(\nabla_{e_{k}} P_{r-1}\right)\left(\nabla_{e_{k}} A\right)-e_{k}\left(S_{r}\right) P_{r-1}\left(\nabla_{e_{k}} A\right)+\left(P_{r-1} \nabla_{e_{k}} A\right)^{2},
\end{aligned}
$$

so that

$$
\begin{aligned}
\sum_{k} \operatorname{tr}\left(T_{k}\right)= & S_{r} \sum_{k} \operatorname{tr}\left[\left(\nabla_{e_{k}} P_{r-1}\right)\left(\nabla_{e_{k}} A\right)\right] \\
& -\sum_{k} \operatorname{tr}\left[e_{k}\left(S_{r}\right) P_{r-1}\left(\nabla_{e_{k}} A\right)\right]+\sum_{k}\left|P_{r-1} \nabla_{e_{k}} A\right|^{2} .
\end{aligned}
$$

Now, lemma 3.2 gives

$$
\sum_{k} \operatorname{tr}\left[e_{k}\left(S_{r}\right) P_{r-1}\left(\nabla_{e_{k}} A\right)\right]=\operatorname{tr}\left[P_{r-1}\left(\nabla_{\nabla S_{r}} A\right)\right]=\left|\nabla S_{r}\right|^{2} .
$$

On the other hand, making $q=0$ in proposition 3.1 one gets

$$
\begin{aligned}
\Delta S_{r} & =L_{r-1}\left(S_{1}\right)+\sum_{k} \operatorname{tr}\left\{\left(\nabla_{e_{k}} P_{r-1}\right)\left(\nabla_{e_{k}} A\right)\right\} \\
& =+c\left[\operatorname{tr}\left(A P_{r-1}\right) n-\operatorname{tr}\left(P_{r-1}\right) S_{1}\right]+\operatorname{tr}\left(A^{2} P_{r-1}\right) S_{1}-\operatorname{tr}\left(A P_{r-1}\right)|A|^{2},
\end{aligned}
$$

so that

(29)

$$
\begin{aligned}
\sum_{k} \operatorname{tr}\left\{\left(\nabla_{e_{k}} P_{r-1}\right)\left(\nabla_{e_{k}} A\right)\right\}= & \Delta S_{r}-L_{r-1}\left(S_{1}\right)-c\left[\operatorname{tr}\left(A P_{r-1}\right) n-\operatorname{tr}\left(P_{r-1}\right) S_{1}\right] \\
& -\operatorname{tr}\left(A^{2} P_{r-1}\right) S_{1}+\operatorname{tr}\left(A P_{r-1}\right)|A|^{2},
\end{aligned}
$$

Substituting (28) and (29) into (27), and then into (26), we finally arrive at

$$
\begin{aligned}
L_{r}\left(S_{r}\right)= & L_{r-1}\left(S_{r+1}\right)+S_{r}\left[\Delta S_{r}-L_{r-1}\left(S_{1}\right)\right] \\
& +\sum_{k}\left|P_{r-1} \nabla_{e_{k}} A\right|^{2}-\left|\nabla S_{r}\right|^{2}-c S_{r}\left[\operatorname{tr}\left(A P_{r-1}\right) n-\operatorname{tr}\left(P_{r-1}\right) S_{1}\right]
\end{aligned}
$$




$$
\begin{aligned}
& -S_{r} \operatorname{tr}\left(A^{2} P_{r-1}\right) S_{1}+S_{r} \operatorname{tr}\left(A P_{r-1}\right)|A|^{2} \\
& +c\left[\operatorname{tr}\left(A P_{r-1}\right) \operatorname{tr}\left(P_{r}\right)-\operatorname{tr}\left(P_{r-1}\right) \operatorname{tr}\left(A P_{r}\right)\right] \\
& +\operatorname{tr}\left(A^{2} P_{r-1}\right) \operatorname{tr}\left(A P_{r}\right)-\operatorname{tr}\left(A P_{r-1}\right) \operatorname{tr}\left(A^{2} P_{r}\right),
\end{aligned}
$$

from where (24) easily follows. In order to get (25), let

$$
\begin{aligned}
T= & \operatorname{tr}\left(A P_{r-1}\right)\left\{S_{r}\left(|A|^{2}-c n\right)-\left[\operatorname{tr}\left(A^{2} P_{r}\right)-c \operatorname{tr}\left(P_{r}\right)\right]\right\} \\
& -\operatorname{tr}\left(A^{2} P_{r-1}\right)\left[\operatorname{tr}\left(A^{2} P_{r-1}\right)-c \operatorname{tr}\left(P_{r-1}\right)\right]
\end{aligned}
$$

and take a basis $\left\{e_{k}\right\}$ of $T_{p} M$ as in the statement of the corollary. Then

$$
\begin{aligned}
T= & \sum_{i} \lambda_{i} S_{r-1}\left(A_{i}\right) S_{r}\left(|A|^{2}-c n\right)+\sum_{i, j} \lambda_{i} S_{r-1}\left(A_{i}\right) S_{r}\left(A_{j}\right)\left(c-\lambda_{j}^{2}\right) \\
& +\sum_{i, j} \lambda_{i}^{2} S_{r-1}\left(A_{i}\right) S_{r-1}\left(A_{j}\right)\left(c-\lambda_{j}^{2}\right) \\
= & \sum_{i} \lambda_{i} S_{r-1}\left(A_{i}\right) \cdot S_{r}\left(|A|^{2}-c n\right) \\
& +\sum_{i} \lambda_{i} S_{r-1}\left(A_{i}\right) \cdot \sum_{j}\left(c-\lambda_{j}^{2}\right)\left[S_{r}\left(A_{j}\right)+\lambda_{i} S_{r-1}\left(A_{j}\right)\right] .
\end{aligned}
$$

Observing that

$$
\begin{aligned}
& S_{r}\left(|A|^{2}-c n\right)+\sum_{j}\left(c-\lambda_{j}^{2}\right)\left[S_{r}\left(A_{j}\right)+\lambda_{i} S_{r-1}\left(A_{j}\right)\right] \\
& \quad=S_{r}\left(|A|^{2}-c n\right)+\sum_{j}\left(c-\lambda_{j}^{2}\right)\left[S_{r}+\left(\lambda_{i}-\lambda_{j}\right) S_{r-1}\left(A_{j}\right)\right] \\
& \quad=\sum_{j}\left(c-\lambda_{j}^{2}\right)\left(\lambda_{i}-\lambda_{j}\right) S_{r-1}\left(A_{j}\right),
\end{aligned}
$$

we get

$$
T=\sum_{i, j} S_{r-1}\left(A_{i}\right) S_{r-1}\left(A_{j}\right) \lambda_{i}\left(\lambda_{i}-\lambda_{j}\right)\left(c-\lambda_{j}^{2}\right)
$$

Doing the same computation as the one above, this time changing $i$ by $j$ from the very beginning, we arrive at

$$
T=\sum_{i, j} S_{r-1}\left(A_{j}\right) S_{r-1}\left(A_{i}\right) \lambda_{j}\left(\lambda_{j}-\lambda_{i}\right)\left(c-\lambda_{i}^{2}\right),
$$

so that 


$$
\begin{aligned}
2 T & =\sum_{i, j} S_{r-1}\left(A_{i}\right) S_{r-1}\left(A_{j}\right)\left(\lambda_{i}-\lambda_{j}\right)\left[\lambda_{i}\left(c-\lambda_{j}^{2}\right)-\lambda_{j}\left(c-\lambda_{i}^{2}\right)\right] \\
& =\sum_{i, j} S_{r-1}\left(A_{i}\right) S_{r-1}\left(A_{j}\right)\left(\lambda_{i}-\lambda_{j}\right)^{2}\left(c+\lambda_{i} \lambda_{j}\right), \\
& =\sum_{i, j} S_{r-1}\left(A_{i}\right) S_{r-1}\left(A_{j}\right)\left(\lambda_{i}-\lambda_{j}\right)^{2} K_{M}\left(\sigma_{i j}\right),
\end{aligned}
$$

where Gauss' equation was used in the last equality.

COROllary 3.4. Let $x: M^{n} \rightarrow \bar{M}_{c}^{n+1}$ be an isometric immersion. Then

$$
\begin{aligned}
L_{1}\left(S_{1}\right)= & \Delta S_{2}+\left\{|\nabla A|^{2}-\left|\nabla S_{1}\right|^{2}\right\}+\operatorname{tr}\left(A P_{1}\right)\left(|A|^{2}-c n\right) \\
& -S_{1}\left[\operatorname{tr}\left(A^{2} P_{1}\right)-c \operatorname{tr}\left(P_{1}\right)\right] .
\end{aligned}
$$

\section{Applications}

From now on, all manifolds are supposed to be connected. Let $x_{1}$ : $\mathbf{S}_{r_{1}}^{n_{1}} \rightarrow \mathbf{R}^{n_{1}+1}$ and $x_{2}: \mathbf{S}_{r_{2}}^{n_{2}} \rightarrow \mathbf{R}^{n_{2}+1}$ be the standard immersions, with second (vector) fundamental forms $\alpha_{1}$ and $\alpha_{2}$. If $r_{1}^{2}+r_{2}^{2}=1$ and $n=n_{1}+n_{2}$, the product immersion $x=\left(x_{1}, x_{2}\right)$ satisfies $x\left(\mathbf{S}_{r_{1}}^{n_{1}} \times \mathbf{S}_{r_{2}}^{n_{2}}\right) \subset \mathbf{S}_{1}^{n+1}$. The Clifford torus $\mathbf{S}_{r_{1}}^{n_{1}} \times \mathbf{S}_{r_{2}}^{n_{2}}$ is the induced immersion $\mathbf{S}_{r_{1}}^{n_{1}} \times \mathbf{S}_{r_{2}}^{n_{2}} \hookrightarrow \mathbf{S}_{1}^{n+1}$.

Orient the Clifford torus via $N=\left(-\frac{r_{2}}{r_{1}} x_{1}, \frac{r_{1}}{r_{2}} x_{2}\right)$, and let $(p, q) \in \mathbf{S}_{r_{1}}^{n_{1}} \times \mathbf{S}_{r_{2}}^{n_{2}}$, $\left\{e_{1}, \ldots, e_{n_{1}}\right\}$ be any orthonormal basis of $T_{p} \mathbf{S}_{r_{1}}^{n_{1}}$ and $\left\{e_{n_{1}+1}, \ldots, e_{n_{1}+n_{2}}\right\}$ be any orthonormal basis of $T_{q} \mathbf{S}_{r_{2}}^{n_{2}}$. Making $E_{i}=\left(e_{i}, 0\right)$ for $1 \leq i \leq n_{1}$, and $E_{i}=\left(0, e_{i}\right)$ for $n_{1}+1 \leq i \leq n_{1}+n_{2}$, we get an orthonormal basis for $T_{(p, q)}\left(\mathbf{S}_{r_{1}}^{n_{1}} \times \mathbf{S}_{r_{2}}^{n_{2}}\right)$. Following [3], the matrix of the second fundamental form $A=A_{N}$ of the Clifford torus with respect to such a basis is given by

$$
A=\left[\begin{array}{cc}
\frac{r_{2}}{r_{1}} I_{n_{1}} & 0 \\
0 & -\frac{r_{1}}{r_{2}} I_{n_{2}}
\end{array}\right] .
$$

Therefore, the fundamental principle of counting allows one to immediately read from this matrix the following expression for the elementary symmetric function $S_{r}$ on the Clifford torus $\mathbf{S}_{r_{1}}^{n_{1}} \times \mathbf{S}_{r_{2}}^{n_{2}}$ in the chosen orientation:

$$
S_{r}=\sum_{0 \leq k \leq r}(-1)^{r-k}\left(\begin{array}{c}
n_{1} \\
k
\end{array}\right)\left(\begin{array}{c}
n_{2} \\
r-k
\end{array}\right)\left(\frac{r_{2}}{r_{1}}\right)^{k}\left(\frac{r_{1}}{r_{2}}\right)^{r-k},
$$

with the convention that $\left(\begin{array}{c}m \\ j\end{array}\right)=0$ whenever $j>m$. Yet another useful relation is true, as asserted by the following 
LEMma 4.1. Let $r_{1}, r_{2}$ be positive real numbers such that $r_{1}^{2}+r_{2}^{2}=1$, and $n_{1}, n_{2}$ be positive integers, $n=n_{1}+n_{2}$. Concerning the Clifford torus $\mathbf{S}_{r_{1}}^{n_{1}} \times \mathbf{S}_{r_{2}}^{n_{2}} \hookrightarrow \mathbf{S}^{n+1}$ one has, for $1 \leq r \leq n$,

$$
\begin{array}{r}
\operatorname{tr}\left(A P_{r-1}\right)\left\{S_{r}\left(|A|^{2}-n\right)-\left[\operatorname{tr}\left(A^{2} P_{r}\right)-\operatorname{tr}\left(P_{r}\right)\right]\right\} \\
-\operatorname{tr}\left(A^{2} P_{r-1}\right)\left[\operatorname{tr}\left(A^{2} P_{r-1}\right)-\operatorname{tr}\left(P_{r-1}\right)\right]=0 .
\end{array}
$$

Proof. Letting $A$ denote the second fundamental form of the standard immersion $\mathbf{S}_{r_{1}}^{n_{1}} \times \mathbf{S}_{r_{2}}^{n_{2}} \hookrightarrow \mathbf{S}^{n+1}$, with respect to $N=\left(-\frac{r_{2}}{r_{1}} x_{1}, \frac{r_{1}}{r_{2}} x_{2}\right)$, it follows from

$$
A^{2}-I=\left(\frac{r_{2}}{r_{1}}-\frac{r_{1}}{r_{2}}\right) A
$$

Therefore, letting $\gamma=\frac{r_{2}}{r_{1}}-\frac{r_{1}}{r_{2}}$, one has for $0 \leq r \leq n$ that

$$
A^{2} P_{r}-P_{r}=\left(A^{2}-I\right) P_{r}=\gamma \cdot A P_{r},
$$

and thus

$$
\begin{aligned}
\operatorname{tr}\left(A P_{r-1}\right) & \left\{S_{r}\left(|A|^{2}-n\right)-\left[\operatorname{tr}\left(A^{2} P_{r}\right)-\operatorname{tr}\left(P_{r}\right)\right]\right\} \\
& -\operatorname{tr}\left(A^{2} P_{r-1}\right)\left[\operatorname{tr}\left(A^{2} P_{r-1}\right)-\operatorname{tr}\left(P_{r-1}\right)\right] \\
= & \operatorname{tr}\left(A P_{r-1}\right)\left\{S_{r} \operatorname{tr}\left(A^{2} P_{0}-P_{0}\right)-\operatorname{tr}\left(A^{2} P_{r}-P_{r}\right)\right\} \\
& -\operatorname{tr}\left(A^{2} P_{r-1}\right) \operatorname{tr}\left(A^{2} P_{r-1}-P_{r-1}\right) \\
= & \operatorname{tr}\left(A P_{r-1}\right)\left[S_{r} \gamma \cdot \operatorname{tr}\left(A P_{0}\right)-\gamma \cdot \operatorname{tr}\left(A P_{r}\right)\right]-\operatorname{tr}\left(A^{2} P_{r-1}\right) \gamma \cdot \operatorname{tr}\left(A P_{r-1}\right) \\
= & \gamma \cdot \operatorname{tr}\left(A P_{r-1}\right)\left[S_{1} S_{r}-(r+1) S_{r+1}\right]-\gamma \cdot \operatorname{tr}\left(A^{2} P_{r-1}\right) \operatorname{tr}\left(A P_{r-1}\right) \\
= & \gamma \cdot \operatorname{tr}\left(A P_{r-1}\right) \operatorname{tr}\left(A^{2} P_{r-1}\right)-\gamma \cdot \operatorname{tr}\left(A^{2} P_{r-1}\right) \operatorname{tr}\left(A P_{r-1}\right)=0 .
\end{aligned}
$$

We now state a slightly modified version of remark 2.1 of [3]:

Lemma 4.2. Let $x: M^{n} \rightarrow \bar{M}_{c}^{n+1}$ be an isometric immersion. Assume that the mean curvature $H$ of $M$ does not change sign, and choose the orientation in such a way that $H \geq 0 .{ }^{1}$ If the scalar curvature $R$ of $M$ satisfies $R \geq c$, then $P_{1} \geq 0$. If $R>c$, then $P_{1}>0$.

Proof. It follows from equation (8) that $R \geq c$ if and only if $S_{2} \geq 0$. Denoting by $\lambda_{1}, \ldots, \lambda_{n}$ the eigenvalues of the second fundamental form $A$ of $x$, one has

\footnotetext{
${ }^{1}$ If $M$ has scalar curvature $R>c$, then $S_{2}>0$. It then follows from $S_{1}^{2}=2 S_{2}+|A|^{2}$ that $H \neq 0$. Therefore, $H$ does not change sign on $M$.
} 


$$
S_{1}^{2}=|A|^{2}+2 S_{2} \geq|A|^{2} \geq \lambda_{i}^{2}
$$

so that $-S_{1} \leq \lambda_{i} \leq S_{1}$. Hence, $S_{1}\left(A_{i}\right)=S_{1}-\lambda_{i} \geq 0$, and $P_{1} \geq 0$. If, at some $p \in M$, one has $S_{1}\left(A_{i}\right)=0$, it follows that $S_{1}=\lambda_{i}$, and (34) gives $S_{2}=0$ and $\lambda_{j}=0$ for all $j \neq i$. Therefore, $S_{1} \geq 0, S_{2}>0 \Rightarrow P_{1}>0$.

Our first result generalizes theorem 3.1 of [3].

THEOREM 4.3. Let $x: M^{n} \rightarrow \mathbf{S}^{n+1}$ be a closed orientable hypersurface of the sphere, with scalar curvature $R \geq 1$. Assume that the mean curvature $H$ of $M$ does not change sign, and orient $M$ in such a way that $H \geq 0$. If $H$ or $R$ is constant on $M$, and

$$
H\left[\operatorname{tr}\left(P_{1}\right)-\operatorname{tr}\left(A^{2} P_{1}\right)\right]+(n-1)(R-1)\left(|A|^{2}-n\right) \geq 0
$$

on $M$, then

(a) $H\left[\operatorname{tr}\left(P_{1}\right)-\operatorname{tr}\left(A^{2} P_{1}\right)\right]+(n-1)(R-1)\left(|A|^{2}-n\right)=0$ on $M$.

(b) $M$ is either totally geodesic or a Clifford torus $\mathbf{S}_{r_{1}}^{n_{1}} \times \mathbf{S}_{r_{2}}^{n_{2}}$, with $r_{1}^{2}+r_{2}^{2}=1$,

$$
\begin{aligned}
& n_{1}, n_{2}>0 \text { and } \beta=\left(\frac{r_{2}}{r_{1}}\right)^{2} \geq 1 \text { satisfying } \\
& \quad n_{1}\left(n_{1}-1\right) \beta^{2}-2 n_{1} n_{2} \beta+n_{2}\left(n_{2}-1\right)=n(n-1)(R-1) \beta .
\end{aligned}
$$

Proof. It follows from (30) that

$$
L_{1}\left(S_{1}\right)=\Delta S_{2}+|\nabla A|^{2}-\left|\nabla S_{1}\right|^{2}+2 S_{2}\left(|A|^{2}-n\right)+S_{1}\left[\operatorname{tr}\left(P_{1}\right)-\operatorname{tr}\left(A^{2} P_{1}\right)\right],
$$

and upon integration over $M$ we get

$$
0=\int_{M}\left\{|\nabla A|^{2}-\left|\nabla S_{1}\right|^{2}+2 S_{2}\left(|A|^{2}-n\right)+S_{1}\left[\operatorname{tr}\left(P_{1}\right)-\operatorname{tr}\left(A^{2} P_{1}\right)\right]\right\} d M .
$$

Now, since $2 S_{2}=n(n-1)(R-1) \geq 0$, lemma 2.5 gives for the case of constant $R$ that $|\nabla A|^{2}-\left|\nabla S_{1}\right|^{2} \geq 0$. Since this inequality is obviously true when $H$ is constant, we thus get

$$
|\nabla A|^{2}-\left|\nabla S_{1}\right|^{2}=0
$$

and

$$
H\left[\operatorname{tr}\left(P_{1}\right)-\operatorname{tr}\left(A^{2} P_{1}\right)\right]+(n-1)(R-1)\left(|A|^{2}-n\right)=0
$$

on $M$. Returning to the expression for $L_{1}\left(S_{1}\right)$, it follows that $L_{1}\left(S_{1}\right)=\Delta S_{2}$. Therefore, whether $H$ or $R$ is constant, we get $\Delta S_{2}=0$, and Hopf's strong maximum principle assures that $S_{2}$ is constant. This in turn gives us $L_{1}\left(S_{1}\right)=0$ in both cases, and by using $|A|^{2}=S_{1}^{2}-2 S_{2}$ we arrive at

$$
\frac{1}{2} L_{1}|A|^{2}=S_{1} L_{1}\left(S_{1}\right)+\left\langle P_{1} \nabla S_{1}, \nabla S_{1}\right\rangle-L_{1}\left(S_{2}\right)=\left\langle P_{1} \nabla S_{1}, \nabla S_{1}\right\rangle .
$$

Integrating again over $M$ gives $\int_{M}\left\langle P_{1} \nabla S_{1}, \nabla S_{1}\right\rangle d M=0$, and the previous lemma gives $\left\langle P_{1} \nabla S_{1}, \nabla S_{1}\right\rangle=0$ on $M$. 
If $S_{2}=0$ (or, equivalently, $R=1$ ) on $M$, it was proved in [3] that $M$ is either totally geodesic or a Clifford torus. Otherwise, $S_{2}>0$ on $M$, and lemma 4.2 gives $P_{1}>0$ on $M$. Thus, $\nabla S_{1}=0$ on $M$, and it follows from (36) that $\nabla A=0$. By theorem 4 of [12], $M^{n}$ is a Clifford torus $\mathbf{S}_{r_{1}}^{n_{1}} \times \mathbf{S}_{r_{2}}^{n_{2}}$, with $r_{1}^{2}+r_{2}^{2}=1$. Finally, lemma 4.1 assures that

$$
n H\left[\operatorname{tr}\left(P_{1}\right)-\operatorname{tr}\left(A^{2} P_{1}\right)\right]+n(n-1)(R-1)\left(|A|^{2}-n\right)=0
$$

on all of these tori, so that the only algebraic condition to be satisfied by $M$ is

$$
2 S_{2}=n(n-1)(R-1) .
$$

It now suffices to refer to expression (32) for $S_{2}$ on Clifford tori.

Example 4.4. We construct some families of nontrivial Clifford tori having constant prescribed scalar curvature $1 \leq R<2$.

When $R \geq 2$, every Clifford torus satisfying (35) also satisfies $\beta>1$. In fact, otherwise one would have from (32) that

$$
n(n-1)(R-1)=n_{1}\left(n_{1}-1\right)-2 n_{1} n_{2}+n_{2}\left(n_{2}-1\right)=\left(n_{1}-n_{2}\right)^{2}-n,
$$

which would give in turn

$$
(n-2)^{2} \geq\left(n_{1}-n_{2}\right)^{2}=n[(n-1)(R-1)+1] \geq n^{2},
$$

a contradiction. For $1 \leq R<2$ there are several families of nontrivial tori (i.e., non-minimal ones) with $\beta=1$ and satisfying (35). For $R=1$, for instance, relation (35) reduces to $\left(n_{1}-n_{2}\right)^{2}=n_{1}+n_{2}$. Any solution $n_{1}=a_{1}, n_{2}=a_{2}$ $\left(a_{1}<a_{2}\right)$ for this equation generates a whole family $n_{1}=a_{k}, n_{2}=a_{k+1}$ of solutions, where the sequence $\left(a_{k}\right)_{k \geq 1}$ satisfies, for $k \geq 1$, the recurrence relation $a_{k+2}=2 a_{k+1}-a_{k}+1$. Since $a_{1}=1, a_{2}=3$ is one solution, we get in this case the family

$$
\mathbf{S}_{1 / \sqrt{2}}^{\left(\begin{array}{c}
k \\
2
\end{array}\right)} \times \mathbf{S}_{1 / \sqrt{2}}^{\left(\begin{array}{c}
k+1 \\
2
\end{array}\right)} \hookrightarrow \mathbf{S}_{1}^{\left(\begin{array}{c}
k+1 \\
3
\end{array}\right)+1}
$$

For $R=3 / 2$, (35) reduces to $\left(n_{1}+n_{2}\right)\left(n_{1}+n_{2}-1\right)=8 n_{1} n_{2}$. Once again, any solution $n_{1}=a_{1}, n_{2}=a_{2}$ for this equation generates a whole family $n_{1}=a_{k}$, $n_{2}=a_{k+1}$ of solutions, where the sequence $\left(a_{k}\right)_{k \geq 1}$ satisfies, for $k \geq 1$, the recurrence relation $a_{k+2}=6 a_{k+1}-a_{k}+1$. Thus, the solution $n_{1}=1, n_{2}=7$ generates a family of Clifford tori having scalar curvature $3 / 2$, the first member of which is $\mathbf{S}_{1 / \sqrt{2}}^{1} \times \mathbf{S}_{1 / \sqrt{2}}^{7} \hookrightarrow \mathbf{S}_{1}^{9}$.

From now on we state and prove our main results, the first of which being a gap theorem that generalizes theorem 5.3.2 of [15] for $(r-1)$-minimal hypersurfaces of the sphere:

THEOREM 4.5. If $x: M^{n} \rightarrow \mathbf{S}^{n+1}$ is a closed oriented hypersurface of the sphere for which $S_{r}=0$, then 


$$
\int_{M} \operatorname{tr}\left(A^{2} P_{r-1}\right)\left[\operatorname{tr}\left(P_{r-1}\right)-\operatorname{tr}\left(A^{2} P_{r-1}\right)\right] d M \leq 0 .
$$

Moreover, if $S_{r+1} \neq 0$ on $M$, then $\operatorname{tr}\left(A^{2} P_{r-1}\right) \operatorname{tr}\left(P_{r-1}\right) \geq \operatorname{tr}\left(A^{2} P_{r-1}\right)^{2}$ on $M$ if and only if $M^{n}$ is a Clifford torus $\mathbf{S}_{r_{1}}^{n_{1}} \times \mathbf{S}_{r_{2}}^{n_{2}}$, with $r_{1}^{2}+r_{2}^{2}=1, n_{1}+n_{2}=n$ and $S_{r}=0$.

Proof. It follows from corollary 3.3 that

$$
0=L_{r-1}\left(S_{r+1}\right)+\sum_{k}\left|P_{r-1} \nabla_{e_{k}} A\right|^{2}+\operatorname{tr}\left(A^{2} P_{r-1}\right)\left[\operatorname{tr}\left(P_{r-1}\right)-\operatorname{tr}\left(A^{2} P_{r-1}\right)\right] .
$$

Integrating over $M$, we get

$$
0=\int_{M} \sum_{k}\left|P_{r-1} \nabla_{e_{k}} A\right|^{2} d M+\int_{M} \operatorname{tr}\left(A^{2} P_{r-1}\right)\left[\operatorname{tr}\left(P_{r-1}\right)-\operatorname{tr}\left(A^{2} P_{r-1}\right)\right] d M,
$$

and hence the first part of the theorem.

To the second part, note firstly that, by lemma 4.1, all Clifford tori $\mathbf{S}_{r_{1}}^{n_{1}} \times \mathbf{S}_{r_{2}}^{n_{2}}$ with $S_{r}=0$ do satisfy $\operatorname{tr}\left(A^{2} P_{r-1}\right)=\operatorname{tr}\left(P_{r-1}\right)$. Conversely, suppose that $\operatorname{tr}\left(A^{2} P_{r-1}\right) \operatorname{tr}\left(P_{r-1}\right) \geq \operatorname{tr}\left(A^{2} P_{r-1}\right)^{2}$ and $S_{r+1} \neq 0$ on $M$. Then

$$
\operatorname{tr}\left(A^{2} P_{r-1}\right) \operatorname{tr}\left(P_{r-1}\right)=\operatorname{tr}\left(A^{2} P_{r-1}\right)^{2}
$$

and

$$
\sum_{k}\left|P_{r-1} \nabla_{e_{k}} A\right|^{2}=0
$$

on $M$, from where it follows that $P_{r-1} \nabla_{e_{k}} A=0$ for all $1 \leq k \leq n$. Since $S_{r}=0$ and $S_{r+1} \neq 0$, item (b) of proposition 2.8 assures that $P_{r-1}$ is invertible, so that $\nabla_{e_{k}} A=0$ for all $1 \leq k \leq n$, or still $\nabla A=0$. Hence, by theorem 4 of [12], $M$ is an open submanifold of a Clifford torus $\mathbf{S}_{r_{1}}^{n_{1}} \times \mathbf{S}_{r_{2}}^{n_{2}}$, with $r_{1}^{2}+r_{2}^{2}=1$ and $n_{1}+n_{2}=n$. Since $M$ is also closed and connected, it follows that $M=$ $\mathbf{S}_{r_{1}}^{n_{1}} \times \mathbf{S}_{r_{2}}^{n_{2}}$.

The nonnegativeness of the sectional curvature $K_{M}$ of $M$ suffices to guarantee that $\operatorname{tr}\left(A^{2} P_{r-1}\right) \operatorname{tr}\left(P_{r-1}\right) \geq \operatorname{tr}\left(A^{2} P_{r-1}\right)^{2}$, as asserted by the following

COROLlary 4.6. Let $x: M^{n} \rightarrow \mathbf{S}^{n+1}$ be a closed, oriented hypersurface of the sphere, with $S_{r}=0$ for some $1 \leq r<n$. If $S_{r+1} \neq 0$ on $M^{n}$, and $M^{n}$ has sectional curvature $K_{M} \geq 0$, then $M^{n}$ is a Clifford torus $\mathbf{S}_{r_{1}}^{n_{1}} \times \mathbf{S}_{r_{2}}^{n_{2}}$, with $r_{1}^{2}+r_{2}^{2}=1$, $n_{1}+n_{2}=n$ and $S_{r}=0$. $p \in M$,

Proof. The proof of corollary 3.3, together with $S_{r}=0$, guarantee that, for 


$$
\operatorname{tr}\left(A^{2} P_{r-1}\right)\left[\operatorname{tr}\left(P_{r-1}\right)-\operatorname{tr}\left(A^{2} P_{r-1}\right)\right]=\frac{1}{2} \sum_{i, j} S_{r-1}\left(A_{i}\right) S_{r-1}\left(A_{j}\right)\left(\lambda_{i}-\lambda_{j}\right)^{2} K_{M}\left(\sigma_{i j}\right),
$$

where $\left\{e_{k}\right\}$ is a moving frame on $M^{n}$, diagonalizing $A$ at $p$, with $A e_{k}=\lambda_{k} e_{k}$ at $p$, and $\sigma_{i j}$ denotes the 2-dimensional subspace of $T_{p} M$ generated by $e_{i}$ and $e_{j}$.

Now, item (b) of proposition 2.8 guarantees that $P_{r-1}$ is definite, so that $S_{r-1}\left(A_{i}\right) S_{r-1}\left(A_{j}\right)>0$ for all $1 \leq i, j \leq n$. Therefore, since $K_{M} \geq 0$, we get

$$
\sum_{i, j} S_{r-1}\left(A_{i}\right) S_{r-1}\left(A_{j}\right)\left(\lambda_{i}-\lambda_{j}\right)^{2} K_{M}\left(\sigma_{i j}\right) \geq 0
$$

on $M^{n}$, or still $\operatorname{tr}\left(A^{2} P_{r-1}\right)\left[\operatorname{tr}\left(P_{r-1}\right)-\operatorname{tr}\left(A^{2} P_{r-1}\right)\right] \geq 0$ on $M^{n}$.

Remark 4.7. In both results above, condition $S_{r+1} \neq 0$ eliminates the possibility of $M^{n}$ being totally geodesic. Moreover, it follows from (31) that, for all Clifford tori $M=\mathbf{S}_{r_{1}}^{n_{1}} \times \mathbf{S}_{r_{2}}^{n_{2}}$, one has

$$
K_{M}\left(\sigma_{i j}\right)=1+\lambda_{i} \lambda_{j}= \begin{cases}1+\frac{r_{2}^{2}}{r_{1}^{2}}, & \text { if } 1 \leq i, j \leq n_{1} \\ 0, & \text { if } 1 \leq i \leq n_{1}<j \leq n \\ 1+\frac{r_{1}^{2}}{r_{2}^{2}}, & \text { if } n_{1}<i, j \leq n\end{cases}
$$

Hence, $K_{M} \geq 0$.

For what comes next, we stress that the ellipticity of the operator $L_{r}$ is equivalent to the positive definiteness of $P_{r}$. In the proof of the next result we use proposition 3.2 of [4], stated below.

Proposition 4.8. Let $M^{n}$ be a closed hypersurface of $\mathbf{R}^{n+1}, \mathbf{H}^{n+1}$ or of an open hemisphere of $\mathbf{S}^{n+1}$, such that $S_{r}>0$ on $M^{n}$ for some $2 \leq r \leq n$. Then, for $1 \leq j \leq r-1$, one has $H_{j}>0$ and $L_{j}$ elliptic on $M^{n}$.

THEOREM 4.9. Let $x: M^{n} \rightarrow \bar{M}_{c}^{n+1}$ be a closed orientable hypersurface of $\bar{M}_{c}^{n+1}$, where $\bar{M}_{c}^{n+1}$ denotes $\mathbf{H}^{n+1}, \mathbf{R}^{n+1}$ or an open hemisphere of $\mathbf{S}^{n+1}$, according to whether $c=-1,0$ or 1 . If $S_{r} \neq 0$ is constant on $M^{n}$ for some $2 \leq r<n$, and $M^{n}$ has sectional curvature $K_{M} \geq 0$, then $M$ is a geodesic hypersphere and $x$ is an embedding.

Proof. First of all, it follows from the hypotheses on $M^{n}$ and $\bar{M}^{n+1}$ the existence of a point $p_{0} \in M$ where all principal curvatures of $x$ have the same sign. Orienting $M^{n}$ in such a way that these curvatures are all positive at $p_{0}$, we get $S_{r}\left(p_{0}\right)>0$, and thus $S_{r}>0$ on $M^{n}$. On the other hand, since $S_{r}$ is constant on $M^{n}$, equation (25) gives, at $p \in M$, 


$$
\begin{aligned}
0= & L_{r-1}\left(S_{r+1}-S_{1} S_{r}\right)+\sum_{k}\left|P_{r-1} \nabla_{e_{k}} A\right|^{2} \\
& +\frac{1}{2} \sum_{i, j} S_{r-1}\left(A_{i}\right) S_{r-1}\left(A_{j}\right)\left(\lambda_{i}-\lambda_{j}\right)^{2} K_{M}\left(\sigma_{i j}\right),
\end{aligned}
$$

where $\left\{e_{k}\right\}$ is a moving frame on a neighborhood of $p$ in $M$, diagonalizing $A$ at $p$, with $A e_{k}=\lambda_{k} e_{k}$, and $\sigma_{i j}$ denotes the 2-dimensional subspace of $T_{p} M$ generated by $e_{i}$ and $e_{j}$.

Now, proposition 4.8 assures the ellipticity of $L_{r-1}$, so that $P_{r-1}$ is positive definite, and $S_{r-1}\left(A_{i}\right)>0$ for all $1 \leq i \leq n$. Therefore,

$$
\sum_{i, j} S_{r-1}\left(A_{i}\right) S_{r-1}\left(A_{j}\right)\left(\lambda_{i}-\lambda_{j}\right)^{2} K_{M}\left(\sigma_{i j}\right) \geq 0,
$$

and equation (40) gives

$$
L_{r-1}\left(S_{r+1}-S_{1} S_{r}\right)+\sum_{k}\left|P_{r-1} \nabla_{e_{k}} A\right|^{2} \leq 0 .
$$

from where $L_{r-1}\left(S_{r+1}-S_{1} S_{r}\right) \leq 0$. Since $M$ is closed and $L_{r-1}$ is elliptic, it follows from Hopf's strong maximum principle ([8]) that $S_{r+1}-S_{1} S_{r}$ is constant on $M$, so that $\sum_{k}\left|P_{r-1} \nabla_{e_{k}} A\right|^{2}=0$. Using again the fact that $P_{r-1}$ is positive definite, we get $\nabla_{e_{k}} A=0$ for $1 \leq k \leq n$, or still $\nabla A=0$ on $M$. Let's now consider three separate cases:

For $c=0$, theorem 4 of [12] assures that, up to isometries of $\mathbf{R}^{n+1}, M$ is an open subset of $\mathbf{S}_{r_{1}}^{n_{1}} \times \mathbf{R}^{n_{2}}$, where $n_{1}+n_{2}=n$ and $n_{1}, n_{2} \geq 0$. Since $M$ is closed (i.e., compact whithout boundary), we have $M^{n}=\mathbf{S}_{r_{1}}^{n}$. The reasoning for $c=-1$ is essentially the same. Suppose now that $M^{n}$ is contained in an open hemisphere of $\mathbf{S}^{n+1}$. It follows again from theorem 4 of [12] that, up to isometries of $\mathbf{S}^{n+1}, M$ is a Clifford torus $\mathbf{S}_{r_{1}}^{n_{1}} \times \mathbf{S}_{r_{2}}^{n_{2}}$, where $n_{1}+n_{2}=n$ and $n_{1}, n_{2} \geq 0$. However, were $n_{1}, n_{2}>0, M$ would not be contained in an open hemisphere of $\mathbf{S}^{n+1}$. Therefore, $\min \left\{n_{1}, n_{2}\right\}=0$, and the closedness of $M$ gives that it is isometric to $\mathbf{S}_{r_{1}}^{n}$, for some $0<r_{1}<1$.

Finally, since in all of the above cases $x: M \rightarrow x(M)$ is a covering of the simply connected space $x(M)$, it follows that $x$ is an embedding.

The hypothesis of $M^{n}$ being contained in an open hemisphere of $\mathbf{S}^{n+1}$ in the above theorem is somewhat restrictive, but can be relaxed once one imposes conditions on $M^{n}$ sufficient to guarantee the ellipticity of $L_{r}$, for some $1 \leq$ $r<n$. The following lemma gives one such set of conditions:

Lemma 4.10. Let $M^{n}$ be an orientable Riemannian manifold, of Ricci curvature Ric $\geq c$, and $x: M^{n} \rightarrow \bar{M}_{c}^{n+1}$ be an isometric immersion. Suppose that the mean curvature $H$ of $M^{n}$ does not change sign, and orient $M$ in such a way that $H \geq 0$. If $S_{r}(p) \neq 0$ for some $2 \leq r \leq n$, then $L_{r-1}$ is elliptic at $p$. 
Proof. Fix $p \in M$ and choose a basis $\left\{e_{k}\right\}$ of $T_{p} M$, diagonalizing $A$ at $p$, with $A e_{k}=\lambda_{k} e_{k}$ for $1 \leq k \leq n$. Gauss' equation gives

$$
\operatorname{Ric}_{p}\left(e_{k}\right)=\frac{1}{n-1} \sum_{i \neq k}\left(c+\lambda_{k} \lambda_{i}\right)=c+\frac{1}{n-1} \lambda_{k}\left(S_{1}-\lambda_{k}\right) \text {. }
$$

Then, $\operatorname{Ric}_{p}\left(e_{k}\right) \geq c$ and $S_{1}(p) \geq 0$ give $0 \leq \lambda_{k} \leq S_{1}(p)$ for $1 \leq k \leq n$. It follows that all summands of $S_{r}(p)$ are nonnegative, so that $S_{r}(p) \geq 0$. If $S_{r}(p) \neq 0$ then $S_{r}(p)>0$, and at least $r$ of the $\lambda_{k}$ are positive, so that, at $p$, at least one of the summands in $S_{r-1}\left(A_{i}\right)$ is positive, for each $1 \leq i \leq n$. Hence, $P_{r-1}$ is positive definite at $p$.

THEOREM 4.11. Let $x: M^{n} \rightarrow \mathbf{S}^{n+1}$ be a complete orientable hypersurface of the unit sphere, with Ricci curvature Ric $\geq 1$. Assume that the mean curvature $H$ of $M$ does not change sign, and orient $M$ in such a way that $H \geq 0$. If, for some $2 \leq r \leq n, S_{r} \neq 0$ is constant on $M$, then

$$
\begin{array}{r}
\operatorname{tr}\left(A P_{r-1}\right)\left\{S_{r}\left(|A|^{2}-n\right)+\left[\operatorname{tr}\left(P_{r}\right)-\operatorname{tr}\left(A^{2} P_{r}\right)\right]\right\} \\
+\operatorname{tr}\left(A^{2} P_{r-1}\right)\left[\operatorname{tr}\left(P_{r-1}\right)-\operatorname{tr}\left(A^{2} P_{r-1}\right)\right] \geq 0,
\end{array}
$$

on $M$ if and only if $M$ is a Clifford torus $\mathbf{S}_{r_{1}}^{n_{1}} \times \mathbf{S}_{r_{2}}^{n_{2}}$, with $n_{1}+n_{2}=n$, and $r_{1}^{2}+r_{2}^{2}=1$.

Proof. When $M$ is a Clifford torus, it follows from lemma 4.1 that (41) becomes an equality. Conversely, suppose that (41) is valid on $M$. Since $S_{r}$ is constant on $M$, one has once more

$$
L_{r-1}\left(S_{r+1}-S_{1} S_{r}\right)+\sum_{k}\left|P_{r-1} \nabla_{e_{k}} A\right|^{2} \leq 0 .
$$

The condition on the Ricci curvature of $M$ assures, via Bonnet-Myers thorem, that $M$ is closed. On the other hand, since $S_{r} \neq 0$ on $M$, the preceeding lemma assures the ellipticity of $L_{r-1}$, and from this point on the reasoning is identical to that of the previous result.

Concerning the non-compact case, we have the following result:

THEOREM 4.12. Let $x: M^{n} \rightarrow \mathbf{R}^{n+1}$ be a complete, non-compact, oriented hypersurface of the Euclidean space, with sectional curvature $K_{M} \geq 0$. If, for some $2 \leq r<n, S_{r} \neq 0$ is constant on $M$, and $S_{1} S_{r}-S_{r+1}$ attains a global maximum on $M$, then $M$ is isometric to $\mathbf{S}_{r_{1}}^{n_{1}} \times \mathbf{R}^{n_{2}}$ for some $r_{1}>0$, where $n_{1}+n_{2}=n$ and $r \leq n_{1}<n$. In particular, if $S_{r+1}=0$ on $M$ and $H$ attains $a$ global maximum on $M$, then $M$ is isometric to $\mathbf{S}_{r_{1}}^{r} \times \mathbf{R}^{n-r}$, for some $r_{1}>0$.

Proof. Since $K_{M} \geq 0$, it follows that $M$ has Ricci curvature Ric $\geq 0$. Moreover, letting $\lambda_{1}, \ldots, \lambda_{n}$ denote the eigenvalues of the second fundamental 
form $A$ of $M$, it also follows from $K_{M} \geq 0$ that, at each point of $M$, one has either $\lambda_{1}, \ldots, \lambda_{n} \geq 0$ or $\lambda_{1}, \ldots, \lambda_{n} \leq 0$. Therefore, $S_{1}$ does not change sign on $M$, for otherwise there would exist $p \in M$ such that $S_{1}(p)=0$, and hence $\lambda_{1}=\cdots=\lambda_{n}=0$ at $p$; this would contradict $S_{r}(p) \neq 0$. The ellipticity of $L_{r-1}$ now follows from lemma 4.10.

Using equation (25), we get at $p \in M$ and for an appropriate frame $\left\{e_{k}\right\}$ that

$$
\begin{aligned}
L_{r-1}\left(S_{1} S_{r}-S_{r+1}\right)= & \sum_{k}\left|P_{r-1} \nabla_{e_{k}} A\right|^{2} \\
& +\frac{1}{2} \sum_{i, j} S_{r-1}\left(A_{i}\right) S_{r-1}\left(A_{j}\right)\left(\lambda_{i}-\lambda_{j}\right)^{2} K_{M}\left(\sigma_{i j}\right) .
\end{aligned}
$$

Since both terms on the right hand side are nonnegative, it follows that $L_{r-1}\left(S_{1} S_{r}-S_{r+1}\right) \geq 0$. The hypothesis of $S_{1} S_{r}-S_{r+1}$ attaining a global maximum on $M$ guarantees, via Hopf's strong maximum principle, that $S_{1} S_{r}-S_{r+1}$ is constant on $M$. It then follows from the above relation that

$$
\sum_{k}\left|P_{r-1} \nabla_{e_{k}} A\right|^{2}=0
$$

on $M$, and thus $\nabla A=0$ on $M$, for $P_{r-1}$ is positive definite.

Finally, applying once more theorem 4 of [12] we get $M^{n}$ isometric to $\mathbf{S}_{r_{1}}^{n_{1}} \times \mathbf{R}^{n_{2}}$, where $n_{1}, n_{2} \geq 0$ and $n_{1}+n_{2}=n$. However, since $M$ is non-compact and $S_{r} \neq 0$ over it, it must happen that $r \leq n_{1}<n$. To finish, note that $S_{r+1}=0$ gives $n_{1}=r$ as the only possible option.

\section{REFERENCES}

[ 1] H. Alencar AND M. Do CARmo, Hypersurfaces with constant mean curvature in spheres, Proc. Amer. Math. Soc. 120 (1994), 1223-1229.

[2] H. Alencar, M. do Carmo and A. G. Colares, Stable hypersurfaces with constant scalar curvature, Math. Z. 213 (1993), 117-131.

[ 3 ] H. Alencar, M. do Carmo and W. Santos, A gap theorem for hypersurfaces of the sphere with constant scalar curvature one, Comment. Math. Helv. 77 (2002), 549-562.

[4] J. L. M. Barbosa AND A. G. Colares, Stability of hypersurfaces with constant $r$-mean curvature, Ann. of Global Anal. Geom. 15 (1997), 277-297.

[ 5 ] S. Y. Cheng And S. T. YaU, Hypersurfaces with constant scalar curvature, Math. Ann. 225 (1977), 195-204.

[6] S. S. Chern, M. P. do Carmo and S. Kobayashi, Compact minimal submanifolds of a sphere with second fundamental form of constant length, Functional analysis and related fields, 1970, 59-75.

[7] M. Dajczer et Al., Submanifolds and isometric immersions, Publish or Perish, Houston, 1990.

[8] D. Gilbarg and N. Trudinger, Elliptic partial differential equations of second order, Springer-Verlag, Berlin, 1983.

[9] G. Hardy, J. E. Littlewood and G. Pólya, Inequalities, Cambridge Univ. Press, Cambridge, 1989. 
[10] J. Hounie AND M. L. Leite, The maximum principle for hypersurfaces with vanishing curvature functions, J. Diff. Geom. 41 (1995), 247-258.

[11] J. Hounie AND M. L. Leite, Two-ended hypersurfaces with zero scalar curvature, Indiana Univ. Math. J. 48 (1999), 867-882.

[12] H. B. Lawson, Local rigidity theorems for minimal hypersurfaces, Ann. of Math. (2) 89 (1969), 187-197.

[13] H. Rosenberg, Hypersurfaces of constant curvature in space forms, Bull. Sc. Math. 117 (1993), 217-239.

[14] R. ReILly, Variational properties of functions of the mean curvatures for hypersurfaces in space forms, J. Diff. Geom. (3) 8 (1973), 465-477.

[15] J. Simons, Minimal varieties in Riemannian manifolds, Ann. of Math. 88 (1968), 62-105.

Departamento de Matemática

Universidade Federal do Ceará

CEP: 60455-760-CAMPUS DO PICI

ForTALEZA, CE

BRAZIL

E-mail: caminha@mat.ufc.br, antonio_caminha@yahoo.com.br 